\title{
Secondary Amyloidosis Presenting as Oral Nodules
}

\author{
Amber Kiyani ${ }^{1}$ \\ Anam Zahid Kiani ${ }^{2}$ \\ Uzair Luqman ${ }^{3}$
}

\author{
BDS, MS, FAAOMP, Dip-ABOMP \\ BDS, MSc \\ BDS, FCPS, FFD RCS, FAOCMP
}

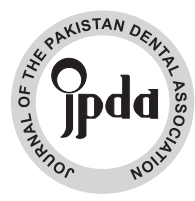

Secondary amyloidosis is a consequence of chronic inflammatory diseases that results in deposition of amyloid in organs disrupting its function. Oral involvement with this process is exceedingly rare. A 63-year-old Pakistani female with a history of rheumatoid arthritis and ankylosing spondylitis was seen in the Oral Medicine Clinics for painful oral nodules that were diagnosed as AA-type, or secondary amyloidosis, following an incisional biopsy. Similar deposits were identified in vital organs on subsequent examination by the patient's physicians. Due to widespread systemic involvement, our patient died within 6-months of her diagnosis.

CONCLUSION: This case serves as a reminder that oral presentations may be the first identifiable signs of systemic diseases. It also emphasizes the importance of multidisciplinary clinical practice, and the lack of it in our country.

KEY WORDS: AA amyloidosis, secondary amyloidosis, oral amyloidosis.

HOW TO CITE: Kiyani A, Kiani AZ, Luqman U. Secondary amyloidosis presenting as oral nodules. J Pak Dent Assoc 2019; 28(4):204-206.

DOI: https://doi.org/10.25301/JPDA.284.204

Received: 28 April 2019, Accepted: 25 July 2019

\section{INTRODUCTION}

$\mathrm{S}$ econdary, or reactive amyloidosis is a type of systemic amyloidosis seen in patients with chronic inflammatory conditions, such as rheumatoid arthritis, inflammatory bowel disease, sarcoidosis, and tuberculosis. It results from proliferation and subsequent deposition of an acute-phase protein called serum amyloid A in tissues. Clinical features are usually slow to develop. The first manifestations are usually proteinuria and renal dysfunction. The severity of renal involvement determines prognosis of the patient. Liver and heart involvement may also be seen. ${ }^{1,2}$

Oral involvement with secondary amyloidosis is exceedingly rare. The most frequently affected site is the tongue. The protein deposition usually results in generalized or nodular macroglossia. More diffuse involvement of the oral cavity has also been reported as yellow nodules, or generalized ulceration. ${ }^{3,4}$

Here we present a case of a 63-year-old Pakistani female who was first diagnosed with secondary amyloidosis following a biopsy of her oral nodules, despite being under the care of multiple physicians for her systemic complaints. Our case emphasizes the need of multidisciplinary practice in Pakistan.

1. Assistant Professor, Department of Oral Medicine, Riphah International University.

2. Registrar, Department of Periodontology, Riphah International University.

3. Consultant Oral Surgeon, Department of Oral and Maxillofacial Surgeon, KRL General Hospital.

Corresponding author: “Dr. Amber Kiyani”’ < akiyani@gmail.com >

\section{CASE REPORT}

A 63-year-old Pakistani female presented to the Oral Medicine Clinics at Riphah International University with a complaint of painful oral nodules present for a "few" weeks. The ulcerated nodules, along with difficulty in swallowing were making food consumption difficult. The patient had lost at least 10 kilograms of weight. Her medical history was positive for rheumatoid arthritis and ankylosing spondylitis, both under treatment with variable doses of corticosteroids for twenty years. She had a recent diagnosis of renal failure that her doctors believed was secondary to her long-term corticosteroid use. She had recently begun complaining of bloody stool and voice changes.

Extraoral examination was unremarkable except for tag-like growths on both eyelids (figure 1). The patient said that they had been present for about two months. Intraoral examination revealed variablysized nodules on the tongue, buccal and labial mucosae (figure 2,3). Some of the tongue nodules were ulcerated explaining the pain associated with eating. No cervical lymphadenopathy or salivary gland enlargement was noted.

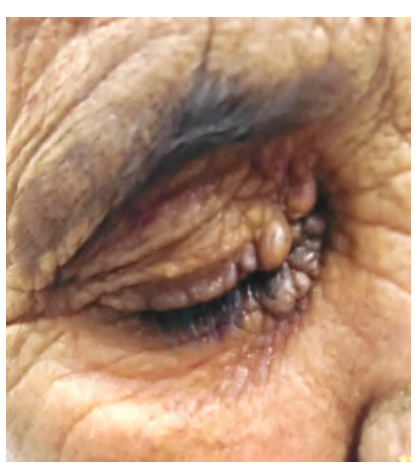

Figure 1: Nodular growths on the upper eyelid 
Considerations in the differential diagnosis at this point included orofacial granulomatosis, oral Crohn's disease and sarcoidosis. All three were ruled out following an incisional biopsy taken from a nodule on the buccal mucosa. It showed amorphous, eosinophilic deposits in the subepithelial and perivascular areas, suggestive of amyloid deposition

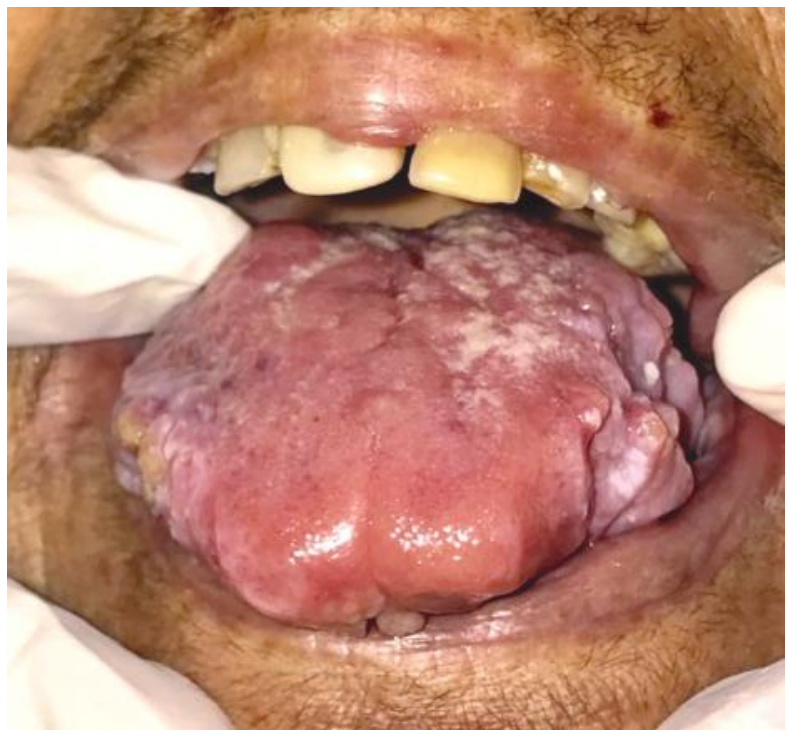

Figure 2: Nodular, ulcerated growths on lateral tongue

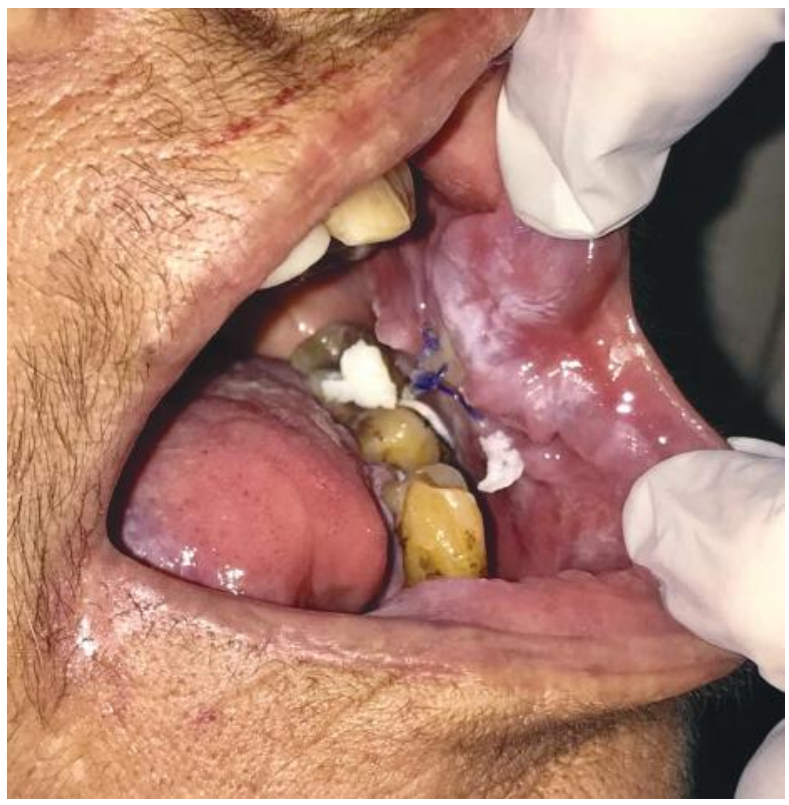

Figure 3: Nodular growths on buccal mucosa, site of incisional biopsy also visible.

(figure 4). The presence of the protein was confirmed by observing characteristic apple-green birefringence on Congo-red stained sections under polarized light. Immunohistochemical staining identified the protein type as AA thus allowing us to classify the disease as secondary amyloidosis.

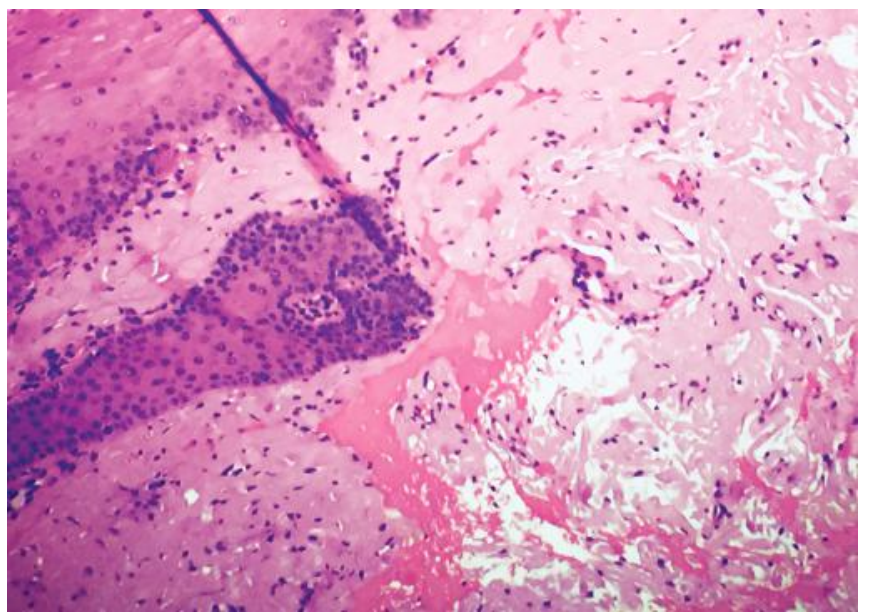

Figure 4: Amorphous eosinophilic deposits in the subepithelial tissue.

The patient was referred to her nephrologist and gastroenterologist. The doctors confirmed deposition of amyloid in the kidney and the anal region. An ear-nosethroat specialist also identified amyloid deposits in the larynx. Due to the widespread involvement the patient was only offered palliative care. She did come back to us for debulking of the tongue and buccal mucosa to facilitate her eating. She died about 6 months after diagnosis.

\section{DISCUSSION}

Our case is an unfortunate account of a delayed diagnosis. Our patient's kidney failure, phonation issues, cutaneous nodules, and gastrointestinal symptoms were all considered and treated as separate issues. By the time a definitive diagnosis was established, there was extensive multisystem involvement and only palliative care could be offered. This case underscores the importance of a multidisciplinary practice approach, and the lack of it in our country.

Amyloidosis is a rare storage disease that results from an abnormal deposition of protein in single, or multiple organs of the body. This gradual buildup of protein causes organ dysfunction and eventual failure. ${ }^{5}$ Common sites of deposition include liver, spleen, kidney, heart and blood vessels. ${ }^{6}$

Amyloidosis is broadly classified into local and systemic types. Systemic amyloidosis is a more severe form of disease that is further categorized into primary, secondary, hemodialysis-associated and heredo-familial types on the basis of etiology. While the causes of hemodialysis-associated and heredo-familial types are straightforward, primary amyloidosis is often a consequence of plasma cell, or B cell neoplasms while secondary amyloidosis occurs in patients with a long-term diagnosis of chronic inflammatory conditions. Each one of these categories is identified by a 
unique protein type; primary by AL (light chain), $\mathrm{AH}$ (heavy chain) or AHL (heavy and light chain), secondary for AA, hemodialysis-associated by $A \beta 2$ microglobulin and hereditary by ATTR. ${ }^{5}$

Oral manifestations of amyloidosis are rare. These include macroglossia and salivary gland hyperplasia. Secondary amyloidosis characteristically presents with tongue enlargement. This enlargement can be diffuse, or nodular like in our case. Other oral site, like buccal mucosa may also be involved. . $^{7,8}$

Diagnosis for oral amyloidosis is established with histopathologic examination. Viewing of sections stained with Congo-red under polarized light remains the gold standard for diagnosis. ${ }^{8}$ Although we were limited by our resources to classify the type of amyloidosis through immunohistochemical staining, more refined techniques involving biomarkers, and genetic sequencing are now available. These offer both diagnostic and prognostic values. ${ }^{5,6}$

Management of amyloidosis requires management of the underlying condition. In case of secondary amyloidosis that means treating the etiological chronic inflammatory condition through immunomodulators. This can slow the deposition of the amyloid protein and enhance longevity. Biomarker investigations have opened up avenues for definition of new therapeutic targets that prevent amyloid fibril deposition and encourage its clearance. ${ }^{5}$

In conclusion, our case is a reminder that oral involvement with secondary amyloidosis, while rare, may be the first identifiable sign of systemic involvement. It also highlights the consequences of a delayed diagnosis and the absence of multidisciplinary practice.

\section{CONFLICT OF INTEREST}

None declared

\section{REFERENCES}

1. Lachmann HJ, Goodman HJ, Gilbertson JA, Gallimore JR, Sabin CA, Gillmore JD, et al. Natural history and outcome in systemic AA amyloidosis. New Eng J Medi. 2007;356:2361-71.

https://doi.org/10.1056/NEJMoa070265

2. Wechalekar AD, Gillmore JD, Hawkins PN. Systemic amyloidosis. Lancet. 2016;387(10038):2641-54. https://doi.org/10.1016/S0140-6736(15)01274-X

3. Shahbaz A, Aziz K, Umair M, Malik ZR, Awan SI, Sachmechi I. Amyloidosis Presenting with Macroglossia. Cureus. 2018;10:e3185.

4. Cengiz MI, Wang HL, Yildiz L. Oral involvement in a case of AA amyloidosis: a case report. J Medi Case Reports. 2010;4:200. https://doi.org/10.1186/1752-1947-4-200
5. Nuvolone M, Merlini G. Systemic amyloidosis: novel therapies and role of biomarkers. Nephrolog, dialysis, transplant: official publication of the Europ Dialysis and Transplant Association - Eur Renal Assoc. 2017;32:770-80.

6. Bustamante JG, Brito D. Amyloidosis. StatPearls. Treasure Island (FL): StatPearls Publishing, StatPearls Publishing LLC.; 2019.

7. Oruba Z, Kaczmarzyk T, Urbanczyk K, Jurczyszyn A, Fornagiel S, Galazka K, et al. Intraoral manifestation of systemic AL amyloidosis with unique microscopic presentation of intracellular amyloid deposition in striated muscles. Polish J path:2018;69:200-04.

https://doi.org/10.5114/pjp.2018.76705

8. Angiero F, Seramondi R, Magistro S, Crippa R, Benedicenti S, Rizzardi C, et al. Amyloid deposition in the tongue: clinical and histopathological profile. Anticancer Res. 2010;30:3009-14. 